\title{
Efeitos da atividade física para a saúde de crianças e adolescentes
}

\section{The effects of physical activity on the health of children and adolescents}

\author{
Paulo Vinícius Carvalho Silva ${ }^{[a]}$, Áderson Luiz Costa Jr. ${ }^{[b]}$
}

[a] Mestre em Processos de Desenvolvimento Humano e Saúde pelo Instituto de Psicologia, Universidade de Brasília (UnB), Brasília, DF - Brasil, e-mail: paulovcs@hotmail.com

[b] Doutor em Psicologia, professor adjunto do Instituto de Psicologia da Universidade de Brasília (UnB), Brasília, DF - Brasil.

\section{Resumo}

A prática regular de atividade física constitui um elemento essencial à promoção da saúde e prevenção de algumas doenças que acometem indivíduos e grupos populacionais. Apesar dos jovens serem a parcela mais ativa da população, os indicadores de sedentarismo crescente têm alertado os profissionais de saúde pública. Para diminuir o sedentarismo, estudos destacam a necessidade dos indivíduos modificarem seus estilos de vida, adquirindo e mantendo ações de promoção da saúde e prevenção de doenças durante todo o curso de vida. Nesse sentido, a atividade física praticada regularmente, pelo menos desde a adolescência, proporciona benefícios físicos e psicológicos considerados preditores da condição de saúde para a vida adulta. Conforme mencionado, os índices de sedentarismo têm constituído uma grande preocupação da saúde pública mundial. Isto pode ser causado, entre outros fatores, pela falta de esclarecimento adequado sobre os efeitos decorrentes da prática de atividade física regular. Sendo assim, o objetivo geral deste ensaio é sintetizar e analisar as informações disponíveis sobre a importância da prática da atividade física para a saúde de crianças e adolescentes, indicando possíveis limitações dos estudos e necessidades de pesquisas futuras. Espera-se que estas informações forneçam subsídios para o desenvolvimento de programas de promoção da atividade física para crianças e adolescentes e incentivem os próprios jovens a buscarem estilos de vida mais saudáveis e ativos. A atividade física deve ser disponibilizada e praticada por todos os jovens, em virtude dos benefícios, a curto e longo prazo, que proporciona à saúde.

Palavras-chave: Atividade física. Promoção da saúde. Prevenção de doenças. Crianças e adolescentes.

\begin{abstract}
The practice of regular physical activity is an essential element to the promotion of health and prevention of some diseases that affect individuals and populations. Although youngsters are the most active population, growing
\end{abstract}


indicators of a sedentary lifestyle have alerted public health professionals. To decrease it, studies highlight the need for individuals to modify their lifestyles, getting and keeping health promotion and disease prevention actions throughout the life course. In this sense, the physical activity performed regularly, at least since adolescence, provides physical and psychological benefits considered predictors of health status into adulthood. As mentioned, the rates of sedentary lifestyles have been a major public health concern worldwide. This can be caused, among other factors, by lack of adequate explanation about the effects of the practice of regular physical activity. Thus, the general objective of this paper is to synthesize and analyze information on the importance of the physical activity practice to the health of children and adolescents, indicating possible limitations of existing studies and future research needs. It is hoped that this information will provide subsidies for the development of physical activity promotion programs in children and adolescents and encourage young people to seek a healthier and more active lifestyle. Physical activity should be available and practiced by all young people due to the benefits provided in the short and long term to health.

Keywords: Physical activity. Health promotion. Disease prevention. Children and adolescents.

\section{Introdução}

Um estilo de vida saudável requer que indivíduos e grupos adquiram e mantenham ações de promoção da saúde e prevenção de doenças durante todo o curso de vida. A Organização Mundial da Saúde (OMS) destaca que a promoção da saúde envolve tanto comportamentos individuais como familiares, bem como políticas públicas eficientes, que protejam as pessoas contra ameaças à saúde e promovam um senso geral de responsabilidade pela maximização da segurança, da vitalidade e do funcionamento integral da pessoa (Jenkins, 2007). hierárquicos:

As ações de prevenção envolvem três níveis

a) primária - com objetivo de evitar que a exposição a riscos (biológicos, ambientais, outros) leve ao desencadeamento de doenças ou traumas;

b) secundária - relacionada à detecção e à intervenção precoces contra condições de doenças, antes que elas se desenvolvam inteiramente;

c) terciária - com objetivo de prevenir complicações avançadas e sequelas de doenças já instaladas, bem como promover a reabilitação do indivíduo tanto quanto possível.

Ou seja, as ações de promoção de saúde e prevenção de doenças pressupõem que o indivíduo é o principal responsável pela mudança de comportamentos de saúde em si próprio e que desempenha papel ativo em relação ao contexto de desenvolvimento da saúde de sua família e comunidade.

A prática regular de atividade física tem sido apontada como um fator relacionado funcionalmente à promoção da saúde dos indivíduos e à prevenção de algumas condições de risco a doenças. Ressalta-se, primeiramente, a necessidade da diferenciação entre os conceitos de atividade física e exercício. De acordo com Caspersen, Powell e Christenson (1985), atividade física consiste em qualquer movimento corporal produzido pelos músculos esqueléticos, resultando em maior gasto energético, quando comparado à taxa metabólica de repouso. Por sua vez, o exercício físico constitui uma subcategoria da atividade física, de caráter planejado, estruturado, repetitivo e intencional, com objetivo de manter ou melhorar um ou mais componentes da aptidão física.

Apesar da relação entre atividade física e estado de saúde, observa-se ainda um alto índice de sedentarismo entre indivíduos, o que tem sido considerada uma das maiores preocupações da saúde pública mundial (Allender, Cowburn \& Foster, 2006; Koezuka et al., 2006; Lopes \& Maia, 2004). Por exemplo, Stone, McKenzie, Welk e Booth (1998) já ressaltavam que uma parcela significativa de crianças e adolescentes não é suficientemente ativa, apontando-se índices de atividade física regular inferiores a 50\%. Jenkins (2007) destaca que o estilo de vida sedentário é o terceiro maior risco de mortalidade e o quinto maior precursor de incapacitação no mundo pós-industrializado.

Em relação aos jovens, o sedentarismo tem constituído grande preocupação para a saúde pública. Estudos apontam maiores índices de sedentarismo entre as meninas (Biddle, Gorely \& Stensel, 2004; 
Stone et al., 1998) e uma tendência à redução do nível de atividade física com o aumento da idade (Duncan, Duncan \& Strycker, 2005; Koezuka et al., 2006; Lopes \& Maia, 2004; Smith \& Green, 2005; Twisk, 2001; Stone et al., 1998), o que justifica o caráter prioritário, atribuído pela saúde pública, nos últimos anos, ao aumento do percentual de jovens que praticam atividade física regularmente (Stone et al., 1998; Trost et al., 2003). A melhora do condicionamento físico, proporcionada pelo aumento da prática de atividade física, é considerada a segunda maneira mais eficaz de reduzir os riscos de doenças futuras (Jenkins, 2007).

Para diminuir o sedentarismo, enfatiza-se a necessidade da mudança de estilo de vida desde a infância (Bois, Sarrazin, Brustad, Trouilloud \& Cury, 2005; Jenkins, 2007; Kohl \& Hobbs, 1998; Lopes \& Maia, 2004; Twisk, 2001). Se praticada regularmente, pelo menos desde a adolescência, a atividade física proporciona benefícios físicos e psicológicos considerados preditores da condição de saúde para a vida adulta (Guerra et al., 2003; Twisk, 2001).

Dunn, Andersen e Jakicic (1998) caracterizam a atividade física como a acumulação diária de pelo menos trinta minutos de atividades, selecionadas pelo indivíduo, considerando eventos de tempo livre, domésticos ou relacionados ao trabalho e que possuem intensidade moderada a vigorosa. Ressalta-se que a atividade física vigorosa é aquela que faz com que o indivíduo transpire, ou respire intensamente, por pelo menos 20 minutos por dia (Biddle et al., 2004).

A carga de atividade física necessária para jovens alcançarem maior capacidade funcional e saúde, conforme descrevem Cavill, Biddle e Sallis (2001), deve incluir uma ação moderada a intensa durante, pelo menos, uma hora por dia. Crianças mais sedentárias deveriam praticar atividade física moderada a intensa durante pelo menos 30 minutos por dia.

Conforme mencionado, os índices de sedentarismo têm constituído uma grande preocupação da saúde pública mundial. Isso pode ser causado, entre outros fatores, pela falta de esclarecimento adequado sobre os efeitos decorrentes da prática de atividade física regular. Sendo assim, o objetivo deste artigo é sintetizar e analisar os efeitos da prática da atividade física sobre o estado de saúde de crianças e adolescentes, indicando limitações dos estudos e necessidades de investigações futuras. Para alcançar seu objetivo, o artigo pretende: a) destacar a importância da atividade física para a promoção da saúde e prevenção de doenças, incluindo o papel do suporte familiar e de amigos para a manutenção da prática de atividade física dos jovens;

b) descrever variáveis preditoras da prática da atividade física e barreiras relacionadas à sua efetivação;

c) discutir aspectos relevantes ao planejamento de intervenções que visem o desenvolvimento de um estilo de vida saudável e mais ativo.

\section{Benefícios decorrentes da prática de atividade física}

A prática regular da atividade física, em geral, pode proporcionar vários benefícios à saúde e ainda constitui uma forma efetiva de prevenção à ocorrência de doenças futuras.

Em relação às crianças, a atividade física desempenha papel fundamental sobre a condição física, psicológica e mental. Conforme descrevem Bois et al. (2005), a prática da atividade física pode aumentar a autoestima, a aceitação social e a sensação de bem-estar entre as crianças. Resultados semelhantes foram obtidos por Savage e Holcomb (citado por Duncan et al., 2005) em relação a adolescentes. $\mathrm{Na}$ idade adulta, estudos ressaltam que a falta de atividade física pode estar relacionada funcionalmente ao desenvolvimento de doenças coronarianas, diabetes mellitus, alguns tipos de câncer, osteoporose, doenças do pulmão e doenças mentais crônicas (Duncan et al., 2005; Sparling, Owen, Lambert \& Haskell, 2000). Nesse sentido, estimativas indicam que $35 \%$ das mortes causadas por diabetes mellitus, $35 \%$ das mortes por doenças cardiovasculares e $32 \%$ das mortes por câncer do intestino poderiam ser prevenidas se as pessoas tivessem uma vida mais ativa e um estilo de vida mais saudável, o que deve ser desenvolvido desde a infância (Powel \& Blair, 1994).

Hohepa, Schofield e Kolt (2006) observaram que jovens estudantes relacionam os efeitos benéficos da prática da atividade física a cinco temas gerais: a) alegria - resultante da socialização com
outros jovens; 
b) realização - com o desenvolvimento pessoal e o reconhecimento social;

c) benefícios físicos - relacionados à aparência, desempenho físico e benefícios à saúde;

d) benefícios psicológicos - relativos ao humor e ao aumento de confiança;

e) fatores ligados a atividades preferenciais, percebendo a atividade física como a melhor opção disponível.

Pesquisas em ciências da saúde apontam que a atividade física regular promove a prevenção de várias doenças. Entre os estudos, ressaltam-se aqueles que apontam a prevenção de:

Obesidade-estudos destacam altosíndices de sobrepeso e obesidade entre crianças e adolescentes norte-americanos (Gunner, Atkinson, Nichols \& Eissa, 2005). Jovens estão expostos ao desenvolvimento de problemas físicos e psicológicos, a curto elongo prazo. Os autores ressaltam a importância de desenvolver estratégias de promoção de saúde que incentivem a adoção de estilos de vida saudáveis. Tais estratégias estão ligadas a quatro fatores:

a) identificação de fatores de risco ligados ao sobrepeso de crianças;

b) superação de barreiras à atividade física;

c) promoção de comportamentos ligados à saúde;

d) estabelecimento de orientações para a prática adequada de atividade física por jovens. A obesidade na infância também apresenta relação funcional com a ocorrência de diabetes tipo II em crianças e adolescentes (Rocchini, 2002).

Distúrbios do sono - há indícios de que a atividade física apresenta uma relação direta com a qualidade do sono (Mello, Boscolo, Esteves \& Tufik, 2005). Essa relação é explicada por duas hipóteses:

a) o aumento da temperatura corporal, decorrente da prática de atividade física, facilita o disparo de mecanismos do sono;

b) o aumento do gasto energético, correspondente à prática de atividade física durante a vigília, aumenta a necessidade de sono para alcançar um balanço energético positivo (Driver \& Taylor, 2000). Um levantamento epidemiológico, realizado na cidade de São Paulo, apontou que $27,1 \%$ de pessoas fisicamente ativas e $72,9 \%$ de pessoas sedentárias se queixavam de insônia. Em relação à sonolência excessiva, $28,9 \%$ de pessoas fisicamente ativas e $71,1 \%$ de pessoas sedentárias se queixavam desse distúrbio (Mello, Fernandes \& Tufik, 2000).

Osteoporose - na idade adulta, altos níveis de atividade física podem prevenir a diminuição de densidade mineral do osso, reduzindo a probabilidade da manifestação de osteoporose (Twisk, 2001).

Saúde mental e aspectos relacionados - estudos realizados com crianças e adolescentes sugerem que a atividade física desempenha uma importante função em relação à autoestima (Twisk, 2001). Entretanto, ainda não há evidências empíricas suficientes para relacionar funcionalmente a prática da atividade física ao maior desenvolvimento social e moral do jovem. Em relação aos adultos, pesquisas apontam que a atividade física, praticada em níveis de intensidade e duração moderados, pode reduzir níveis de estresse (Twisk, 2001). A prática regular de atividade física, pela população em geral, também está associada à ausência ou à reduzida ocorrência de sintomas depressivos ou de ansiedade (Mello et al., 2005).

Doenças cardiovasculares - diversas pesquisas ressaltam a importância da atividade física para a prevenção de doenças cardiovasculares. Segundo Twisk (2001), a maior parte dos estudos sobre a relação entre a atividade física e a saúde cardiovascular de crianças e adolescentes analisa apenas os fatores de risco, como os níveis de gordura no sangue, entre praticantes e não praticantes de atividade física. Alguns estudos populacionais apontam para a relação entre o nível de atividade física e a diminuição do risco de doenças cardiovasculares; indivíduos que relataram praticar níveis mais intensos de atividade física foram justamente aqueles que apresentaram o menor risco para transtornos cardiovasculares (Dunn et al., 1998). No mesmo sentido, Ciolac e Guimarães (2004) apontam que a prática regular de atividade física está associada à manutenção de 
menores índices de pressão arterial em repouso, relação válida a todas as faixas etárias.

Apesar dos inúmeros benefícios à saúde, associados à atividade física regular, alguns estudos mostram que eles não constituem o principal motivo para a sua prática (Allender et al., 2006), destacandose, entre outros fatores, a maior oportunidade para interação, suporte social e obtenção de satisfação pessoal com a atividade. Em relação às crianças, os estudos ressaltam a importância do suporte fornecido pela família como variável mantenedora da prática da atividade física.

\section{Influência da família e dos amigos na prática de atividade física}

Segundo Lee (citado por Duncan et al., 2005), o suporte social oferecido à prática da atividade física tende a variar conforme a idade dos indivíduos praticantes. Em relação à prática da atividade física de crianças e adolescentes, por exemplo, os amigos parecem desempenhar um papel tão relevante quanto a família. Duncan et al. (2005) afirmam que o suporte proporcionado por amigos pode exercer diferentes funções:

a) integração social ou companhia, que corresponde aos momentos em que as pessoas praticam a atividade física juntas;

b) suporte emocional, tal como incentivo e apoio moral, por exemplo;

c) suporte informacional;

d) suporte instrumental, representado pela provisão dos recursos necessários à prática da atividade. Já o suporte familiar constitui uma importante fonte emocional, informacional e instrumental. Além disso, os pais podem representar modelos relevantes, relacionados à prática de atividade física, às crianças e jovens (Kohl \& Hobbs, 1998; StuckyRopp \& DiLorenzo, 1993).

Ou seja, além de constituírem variáveis mantenedoras da prática de atividade física dos filhos, os que praticam regularmente tais atividades parecem exercer maior magnitude de influência (e de mediação) sobre as crianças e jovens.

\section{A relaçáo entre comportamentos sedentários e a prática reduzida de atividade física entre crianças e adolescentes}

As atividades consideradas sedentárias são aquelas realizadas em momentos de desocupação. Entre elas, podemos incluir: assistir televisão, utilizar o computador, realizar alguma leitura e jogar videogame. A relação entre comportamentos sedentários e a diminuição da prática de atividade física é investigada por alguns estudos (Koezuka et al., 2006; Sothern, Loftin, Suskind, Udall \& Blecker, 1999). Segundo Biddle et al. (2004), os estudos apontam para uma correlação positiva entre comportamentos sedentários e reduzida prática de atividades físicas, não sendo possível afirmar que o comportamento sedentário seja a principal variável relacionada funcionalmente à pouca atividade física praticada por determinados indivíduos e grupos. Nesse mesmo sentido, Kohl e Hobbs (1998) destacam que a quantidade de tempo gasta pelas crianças com televisão e videogame não pode ser considerada a causa da redução da atividade física pelos jovens. No entanto, o acesso facilitado à televisão e ao videogame diminui a oportunidade das crianças praticarem atividades físicas.

Zabinski, Norman, Sallis, Calfas e Patrick (2007) enfatizam a importância de que os programas de promoção de saúde, destinados aos jovens, incluam metas de redução da quantidade de tempo gasto em atividades sedentárias, tais como programas de televisão e internet. Para isso, sugerem a necessidade da autoeficácia dos jovens, do envolvimento dos pais e da modificação estrutural e funcional dos ambientes domésticos.

Embora os pesquisadores em geral destaquem a importância do desenvolvimento de programas de promoção à prática de atividade física, diversos aspectos devem ser considerados para a implementação de medidas mais efetivas.

\section{Promoçáo da atividade física regular para crianças e adolescentes}

Para o desenvolvimento de alguma medida eficaz de promoção da atividade física entre crianças e adolescentes, é necessário o conhecimento sistemático de alguns elementos: 
a) particularidades da prática de atividade física de crianças e adolescentes;

b) variáveis preditoras da prática de atividade física;

c) barreiras à prática da atividade física.

Essas informações podem fornecer subsídios indispensáveis ao planejamento de programas efetivos de promoção de atividade física aos jovens.

\section{Características e variáveis preditoras da prática de atividade física entre crianças e adolescentes}

Muitas variáveis devem ser consideradas quando se discute as características mais adequadas das atividades físicas disponibilizadas a crianças e adolescentes. As diferentes estações climáticas, por exemplo, exercem influência diversificada sobre a prática de atividade física das crianças (Ross, Dotson, Gilbert \& Katz, 1985). O nível de atividade física é maior durante o verão, cai durante o outono, é menor durante o inverno e volta a crescer na primavera. Ressalta-se, ainda, que as atividades realizadas pelos meninos tendem a ser diferentes das atividades das meninas e variam conforme a faixa etária (Corbin, 2002).

Stucky-Ropp e DiLorenzo (1993) realizaram um estudo com crianças e suas respectivas mães, com o objetivo de investigar fatores que podem influenciar o nível de atividade física praticado pelas crianças. As respostas das crianças apontaram variáveis preditoras da prática de atividade física:

a) autoeficácia da atividade física, relativa ao julgamento da criança acerca do quanto ela própria é tão boa em comparação com outras crianças que praticam a mesma atividade;

b) modelação direta exercida pelos pais, referente à frequência com que os pais realizam a atividade física com os filhos;

c) suporte oferecido pelos amigos e pela familia, que consiste na frequência com que a família encoraja e se exercita com a criança;

d) satisfação pessoal em relação à atividade física; e) equipamento doméstico disponível, relativo ao número e à qualidade de objetos domésticos que se relacionam aos exercícios;

f) conhecimento sobre atividade física;

g) indicadores negativos de atividade física, representados pelo número de horas gastas pelas crianças assistindo televisão ou jogando videogame.

Devem-se destacar, também, comportamentos promotores de saúde que contribuem para a participação dos jovens na atividade física:

a) envolvimento da família nas atividades;

b) provisão de modelos, pelos pais, relacionados à prática de atividade física;

c) envolvimento das crianças em atividades novas e variadas com pouco tempo de duração (Gunner et al., 2005).

\section{Barreiras à prática de atividade física de crianças e adolescentes}

A prática da atividade física pode encontrar alguns obstáculos de naturezas diversas. Allender et al. (2006) sinalizam que as barreiras à prática da atividade física são compostas principalmente por três aspectos: custos elevados, ambientes sem segurança e pouco acesso às facilidades existentes. Neumark-Sztainer (citado por Gunner et al., 2005) também enumera algumas dificuldades à prática da atividade física:

a) preocupação dos pais em relação à segurança na vizinhança, impedindo que os filhos realizem mais atividades ao ar livre;

b) demandas relacionadas ao trabalho dos pais, que impedem o envolvimento deles em atividades de lazer;

c) condições estruturais da vizinhança que não possibilitam a prática de caminhada;

d) concorrência de programas de computador e de televisão, que incentivam a prática de atividades sedentárias;

e) percepção de que as atividades relacionadas à perda de peso possuem um 
custo mais elevado que o benefício a ser obtido.

Um estudo sobre as barreiras à prática da atividade física, realizado com jovens estudantes, apontou seis variáveis principais:

a) ambientes que estimulam a prática de atividades sedentárias;

b) influência adversa de amigos;

c) baixa estrutura de oportunidades ligadas à atividade física;

d) obstáculos físicos;

e) baixo nível de motivação;

f) falta de tempo.

Destaca-se que tais barreiras foram referidas como presentes em diferentes contextos de desenvolvimento, tais como a família e a escola (Hohepa et al., 2006).

\section{Estratégias e programas de promoçáo de atividade física a crianças e adolescentes}

Segundo Sothern et al. (1999), as estratégias de promoção de exercício devem incluir a identificação de fatores culturais, individuais e da sociedade, que afetam o desenvolvimento e a manutenção, a longo prazo, do aumento dos padrões de atividade física. Um aspecto importante é que tais estratégias devem respeitar particularidades e necessidades de seus respectivos públicos-alvo.

Hohepa et al. (2006) realizaram um estudo com o objetivo de investigar a percepção de estudantes adolescentes sobre os diversos contextos relacionados à atividade física e ideias de estratégias de promoção da atividade. Os jovens mencionaram estratégias relacionadas a três categorias gerais:

a) disponibilidade e acesso, relativos à criação de novas atividades, suporte logístico e modificação/atualização do currículo de educação física nas escolas;

b) suporte da família e dos amigos, que consiste no envolvimento e incentivo das pessoas, incluindo orientações parentais;

c) responsabilidade pessoal, que corresponde ao desenvolvimento de motivação e autoconfiança.
Em relação às ações que promovem o aumento da prática de atividade física entre crianças e adultos, destacam-se estratégias que possibilitem o desenvolvimento de um estilo de vida saudável pelos próprios indivíduos. Dunn et al. (1998) apontam efeitos benéficos, a longo prazo, decorrentes do aumento da atividade física moderada e da redução de atividades sedentárias. Esse tipo de intervenção também mostrou resultados positivos em relação à prevenção de doenças cardiovasculares, reduzindo os índices de gordura corporal e pressão sanguínea.

A realização de mudanças estruturais sobre o ambiente, com a finalidade de promover a prática da atividade física, também é ressaltada em alguns estudos. Foster e Hillsdon (2004), por exemplo, reconhecem que o ambiente influencia determinados comportamentos de saúde. No entanto, os efeitos da mudança do ambiente sobre o aumento da atividade física ainda são pouco esclarecedores. Devem ser realizadas mais pesquisas com o objetivo de verificar o impacto que as mudanças estruturais do ambiente exercem sobre a prática de atividade física entre indivíduos e grupos. Nessa direção, Kohl e Hobbs (1998) sugerem que a modificação do ambiente escolar também interfere sobre o nível de atividade física praticada por crianças e adolescentes. Programas escolares e esportivos, com a finalidade de promover a atividade física, devem ser disponibilizados a todos os segmentos da população, contribuindo para o desenvolvimento de um estilo de vida saudável e mais ativo.

\section{Consideraçóes finais}

Embora os jovens ainda constituam a parcela mais ativa da população, observa-se uma gradativa redução da prática regular de atividades físicas, em parte, decorrente dos avanços tecnológicos do mundo atual. $\mathrm{O}$ acesso facilitado ao microcomputador, o desenvolvimento de videogames mais interativos e instigantes, as atrações disponibilizadas por canais de televisão e pela internet, bem como a percepção de falta de segurança pública, retratada diariamente pela mídia, constituem fatores que contribuem para a mudança em relação às formas de lazer de crianças e jovens (Koezuka et al., 2006). Atualmente, parece haver grande valorização e investimento em formas sedentárias de divertimento e um menor envolvimento de jovens com atividades físicas ao ar livre. 
Ou seja, as mudanças sociais e culturais têm afetado a participação dos jovens em atividades físicas.

Observa-se que alguns estudos não caracterizam suficientemente o conceito de atividade física, utilizando-o como sinônimo de exercício físico. Tal confusão conceitual pode acarretar maiores dificuldades de entendimento para a população sobre as diferenças e a relevância da prática regular de atividades físicas. É necessário esclarecer que a atividade física tem um significado mais amplo, englobando os exercícios físicos, que são atividades planejadas, estruturadas e repetitivas (Caspersen et al., 1985).

Os estudos que investigam o impacto da atividade física sobre a saúde das crianças enfrentam dificuldades quanto à definição da quantidade de atividade física necessária aos jovens. Observa-se grande variabilidade de métodos de mensuração, o que dificulta a sistematização de comparações entre os resultados obtidos por diferentes estudos. Assim, aponta-se a necessidade de maior padronização das formas de avaliação, incluindo-se, por exemplo, a construção e validação de instrumentos que pudessem ser utilizados pela maior parte dos pesquisadores.

Acrescenta-se também que os estudos sobre os efeitos da atividade física na infância e adolescência enfatizam benefícios proporcionados à promoção da saúde e prevenção de doenças. Sendo assim, há uma grande preocupação em incentivar crianças e adolescentes a, desde cedo, desenvolverem estilos de vida saudável e que valorizem as atividades físicas. Os jovens devem ser claramente informados a respeito dos benefícios relacionados à saúde, a curto e longo prazo, da prática regular de atividades físicas, assumindo responsabilidade direta pelo desenvolvimento dos seus próprios comportamentos de saúde. Também devem ser desenvolvidos programas multiprofissionais de intervenção, que incentivem a aquisição e a manutenção de comportamentos de saúde por toda a população.

Além de serem destinados a todos os segmentos da população, os programas de promoção $\mathrm{da}$ atividade física devem envolver os diversos sistemas que se relacionam aos jovens: família, escola e comunidade. O suporte emocional e logístico que esses sistemas oferecem aos jovens é fundamental ao envolvimento motivacional na atividade física. A prática da atividade física deve ser considerada prioridade tanto ao jovem quanto aos seus cuidadores (Duncan et al., 2005).
Um corpo sistemático de conhecimento sobre os efeitos da atividade física a longo prazo poderia ser obtido com uma maior quantidade de estudos longitudinais. Ou seja, indivíduos e grupos poderiam ser acompanhados, da infância à idade adulta, verificando-se os efeitos da prática regular de atividades físicas sobre diversos indicadores de saúde.

As medidas de incentivo à prática de atividade física devem, no entanto, observar as necessidades psicossociais de crianças e adolescentes. As atividades devem despertar o interesse dos jovens e serem adequadas ao nível de desenvolvimento físico e cognitivo, bem como às suas preferências. Além disso, devem ser respeitadas as diferenças entre atividades realizadas por crianças e adultos e, também, a variabilidade cultural entre meninos e meninas.

Em perspectiva semelhante, destaca-se que as recomendações, correspondentes à quantidade e à intensidade da atividade física a ser praticada, podem variar segundo o estado de saúde e a faixa etária dos indivíduos. Ou seja, o foco da atividade física deve ser o indivíduo, seus respectivos interesses, potencialidades e limitações. A atividade física precisa ser percebida como atraente, para aumentar a probabilidade da adesão. Esse é um dos desafios a serem superados pela família, pela escola e pela comunidade, isto é, constituir um ambiente de cuidados que atenda às necessidades de crianças e adolescentes, incentivando-os à prática de atividades físicas como um requisito básico ao desenvolvimento.

Os programas de promoção de atividade física devem estar atentos às características do público-alvo. Sendo assim, estudos epidemiológicos podem contribuir com o delineamento do perfil populacional relacionado a um estilo de vida saudável e ligado à atividade física. Tais informações são fundamentais ao planejamento de programas de incentivo à prática regular de atividades físicas. Ressalta-se, também, a necessidade da publicação de mais estudos sobre os efeitos da prática de atividades físicas entre crianças e adolescentes em periódicos de circulação nacional. A maior parte dos artigos identificados por este trabalho encontra-se publicada em periódicos de circulação internacional.

Os artigos analisados se concentram, quase que exclusivamente, na relação entre a atividade física, a promoção da saúde e a prevenção primária. Entretanto, a atividade física regular pode estar relacionada à prevenção secundária e terciária. Em relação à prevenção secundária, a atividade física pode representar uma 
forma de intervenção precoce em relação a alguma doença que está se desenvolvendo. No caso da prevenção terciária, após a doença ter se desenvolvido, a atividade física regular, caso sejapermitida, pode prevenir algumas complicações e promover a reabilitação física e social dos indivíduos (Jenkins, 2007). Atualmente, a atividade física encontra-se presente em vários programas de reabilitação para indivíduos com doenças cardiovasculares, por exemplo (Dunn et al., 1998).

Atualmente, nota-se o surgimento, cada vez maior, de projetos sociais cujo foco principal é a atividade física como promotora de desenvolvimento. Nessas ações, jovens têm acesso a condições e oportunidades que provavelmente não teriam em outra situação em razão da falta de ambientes adequados. Sugere-se que o Estado e as autoridades competentes priorizem investimentos em projetos de construção de quadras poliesportivas, áreas de lazer e no aproveitamento desses espaços para a realização de programas diversificados com a comunidade local.

\section{Referências}

Allender, S., Cowburn, G., \& Foster, C. (2006). Understanding participation in sport and physical activity among children and adults: A review of qualitative studies. Health Education Research, 21(6), 826-835.

Biddle, S. J. H., Gorely, T., \& Stensel, D. J. (2004). Healthenhancing physical activity and sedentary behavior in children and adolescents. Journal of Sports Sciences, 22(8), 679-701.

Bois, J. E., Sarrazin, P. G., Brustad, R. J., Trouilloud, D. O., \& Cury, F. (2005). Elementary schoolchildren's perceived competence and physical activity involvement: The influence of parents' role modeling behaviors and perceptions of their child's competence. Psychology of Sport and Exercise, 6(4), 381-397.

Caspersen, C. J., Powell, K. E., \& Christenson, G. M. (1985). Physical activity, exercise, and physical fitness: Definitions and distinctions for healthrelated research. Public Health Reports, 100(2), 126-131.

Cavill, N., Biddle, S., \& Sallis, J. F. (2001). Health-enhancing physical activity for young people: Statement of the United Kingdom expert consensus conference. Pediatric Exercise Science, 13, 12-25.
Ciolac, E. G., \& Guimarães, G. V. (2004). Exercício físico e síndrome metabólica. Revista Brasileira de Medicina do Esporte, 10(4), 319-324.

Corbin, C. B. (2002). Physical activity for everyone: What every physical educator should know about promoting lifelong physical activity. Journal of Teaching in Physical Education, 21(2), 128-144.

Driver, H. S., \& Taylor, S. R. (2000). Exercise and sleep. Sleep Medicine Reviews, 4(4), 387-402.

Duncan, S. C., Duncan, T. E., \& Strycker, L. A. (2005). Sources and types of social support in youth physical activity. Healthy Psychology, 24(1), 3-10.

Dunn, A. L., Andersen, R. E., \& Jakicic, J. M. (1998). Lifestyle physical activity interventions: History, short and long-term effects, and recommendations. American Journal of Preventive Medicine, 15(4), 398-412.

Foster, C., \& Hillsdon, M. (2004). Changing the environment to promote health-enhancing physical activity. Journal of Sports Sciences, 22(8), 755-769.

Guerra, S., Oliveira, J., Ribeiro, J. C., Teixeira-Pinto, A., Duarte, J. A., \& Mota, J. (2003). Relação entre a atividade física regular e a agregação de fatores de risco biológicos das doenças cardiovasculares em crianças e adolescentes. Revista Brasileira de Saúde Materno-Infantil, 3(1), 9-15.

Gunner, K. B., Atkinson, P. M., Nichols, J., \& Eissa, M. A. (2005). Health promotion strategies to encourage physical activity in infants, toddlers, and preschoolers. Journal of Pediatric Health Care, 19(4), 253-258.

Hohepa, M., Schofield, G., \& Kolt, G. S. (2006). Physical activity: What do high school students think? Journal of Adolescent Health, 39(3), 328-336.

Jenkins, C. D. (2007). Construindo uma saúde melhor: Um guia para a mudança de comportamento. Porto Alegre: Artmed.

Koezuka, N., Koo, M., Alisson, K. R., Adlaf, E.M., Dwyer, J. J. M., Faulkner, G., et al. (2006). The relationship between sedentary activities and physical inactivity among adolescents: Results form the canadian community health survey. Journal of Adolescent Health, 39(4), 515-522.

Kohl, H. W., \& Hobbs, K. E. (1998). Development of physical activity behaviors among children and adolescents. Pediatrics, 101(3 Pt 2), 554-569. 
Lopes, V. P., \& Maia, J. A. R. (2004). Actividade física nas crianças e jovens. Revista Brasileira de Cineantropometria \& Desempenho Humano, 6(1), 82-92.

Mello, M. T., Fernandes, A. C., \& Tufik, S. (2000). Levantamento epidemiológico da prática de atividade física na cidade de São Paulo. Revista Brasileira de Medicina do Esporte, 6(4), 119-124.

Mello, M. T., Boscolo, R. A., Esteves, A. M., \& Tufik, S. (2005). O exercício físico e os aspectos psicobiológicos. Revista Brasileira de Medicina do Esporte, 11(3), 203-207.

Powel, K. E., \& Blair, S. N. (1994). The public health burden of sedentary living habits: Theoretical but realistic estimates. Medicine and Science in Sports and Exercise, 26(7), 851-856.

Rocchini, A. P. (2002). Childhood obesity and a dibetes epidemic. The New England Journal of Medicine, 346(11), 854-855.

Ross, J. G., Dotson, C. O., Gilbert, G. G., \& Katz, S. J. (1985). After physical education: Physical activity outside of school physical education programs. Journal of Physical Education, Recreation and Dance, 56(1), 35-39.

Smith, A., \& Green, K. (2005). The place of sport and physical activity in young people's lives and its implications for health: Some sociological comments. Journal of Youth Studies, 8(2), 241-253.

Sothern, M. S., Loftin, M., Suskind, R. M., Udall, J. N., \& Blecker, U. (1999). The health benefits of physical activity in children and adolescents: Implications for chronic disease prevention. European Journal of Pediatry, 158(4), 271-274.

Sparling, P. B., Owen, N., Lambert, E. V., \& Haskell, W. L. (2000). Promoting physical activity: The new imperative for public health. Health Education Research, 15(3), 367, 376.

Stone, E. J., McKenzie, T. L., Welk, G. J., \& Booth, M. L. (1998). Effects of physical activity in youth: Review and synthesis. American Journal of Preventive Medicine, 15(4), 298-315.

Stucky-Ropp, R. C., \& DiLorenzo, T. M. (1993). Determinants of exercise in children. Preventive Medicine, 22(6), 880-889.
Trost, S. G., Sallis, J. F., Pate, R. R., Freedson, P. S., Taylor, W. C., \& Dowda, M. (2003). Evaluating a model of parental influence on youth physical activity. American Journal of Preventive Medicine, 25(4), 277-282.

Twisk, J. W. R. (2001). Physical activity guidelines for children and adolescents. Sports Medicine, 31, 617-627.

Zabinski, M. F., Norman, G. J., Sallis, J. F., Calfas, K. J., \& Patrick, K. (2007). Patterns of sedentary behavior among adolescents. Health Psychology, 26(1), 113-120.

Recebido: 02/02/2010

Received: 02/02/2010

Aprovado: 30/06/2010

Approved: 06/30/2010 